\title{
Influence of intersublattice coupling on the terahertz nutation spin dynamics in antiferromagnets
}

\author{
Ritwik Mondal $\oplus^{1,2, *}$ and Peter M. Oppeneer $\oplus^{1}$ \\ ${ }^{1}$ Department of Physics and Astronomy, Uppsala University, Box 516, SE-75120 Uppsala, Sweden \\ ${ }^{2}$ Department of Spintronics and Nanoelectronics, Institute of Physics of the Czech Academy of Sciences, \\ Cukrovarnická 10, CZ-162 00 Praha 6, Czech Republic
}

(Received 15 July 2021; accepted 17 August 2021; published 2 September 2021)

\begin{abstract}
Spin-nutation resonance has been well explored in one-sublattice ferromagnets. Here, we investigate the spin nutation in two-sublattice antiferromagnets as well as, for comparison, ferrimagnets with inter- and intrasublattice nutation coupling. In particular, we derive the susceptibility of the two-sublattice magnetic system in response to an applied external magnetic field. To this end, the antiferromagnetic and ferrimagnetic (sub-THz) precession and $\mathrm{THz}$ nutation resonance frequencies are calculated. Our results show that the precession resonance frequencies and effective damping decrease with intrasublattic nutation coupling, while they increase with intersublattice nutation in an antiferromagnet. However, we find that the THz nutation resonance frequencies decrease with both the intra- and intersublattice nutation couplings. For ferrimagnets, conversely, we calculate two nutation modes with distinct frequencies, unlike antiferromagnets. The exchangelike precession resonance frequency of ferrimagnets decreases with intrasublattice nutation coupling and increases with intersublattice nutation coupling, as antiferromagnets, but the ferromagneticlike precession frequency of ferrimagnets is practically invariant to the intra- and intersublattice nutation couplings.
\end{abstract}

DOI: 10.1103/PhysRevB.104.104405

\section{INTRODUCTION}

Efficient spin manipulation at ultrashort timescales holds promise for applications in future magnetic memory technology [1-5]. Introduced by Landau and Lifshitz, the time evolution of magnetization $\boldsymbol{M}(\boldsymbol{r}, t)$ can be described by the phenomenological Landau-Lifshitz-Gilbert (LLG) equation of motion, which reads [6-9]

$$
\dot{\boldsymbol{M}}=-\gamma(\boldsymbol{M} \times \boldsymbol{H})+\frac{\alpha}{M_{0}}(\boldsymbol{M} \times \dot{\boldsymbol{M}}),
$$

with the gyromagnetic ratio $\gamma$, constant magnetization amplitude $M_{0}$, and Gilbert damping parameter $\alpha$. The LLG equation consists of the precession of spins around a field $\boldsymbol{H}$ and transverse damping that aligns the spins towards the field direction. While the spin precessional motion can be explained by Zeeman-like field-spin coupling, there are several fundamental and microscopic mechanisms leading to Gilbert damping [10-22].

When one approaches the femtosecond regime, however, the spin dynamics can not only be described by the traditional LLG dynamical equation of motion [23,24], but it has to be supplemented by a fast dynamics term due to magnetic inertia [25-28]. Essentially, the inclusion of magnetic inertia

*mondal@fzu.cz

Published by the American Physical Society under the terms of the Creative Commons Attribution 4.0 International license. Further distribution of this work must maintain attribution to the author(s) and the published article's title, journal citation, and DOI. Funded by Bibsam. leads to a spin nutation at ultrashort timescales and can be described by a torque due to a double time derivative of the magnetization, i.e., $\boldsymbol{M} \times \ddot{\boldsymbol{M}}[27,29]$. The inertial LLG (ILLG) equation of motion has the form

$$
\dot{\boldsymbol{M}}=-\gamma(\boldsymbol{M} \times \boldsymbol{H})+\frac{\alpha}{M_{0}}(\boldsymbol{M} \times \dot{\boldsymbol{M}})+\frac{\eta}{M_{0}}(\boldsymbol{M} \times \ddot{\boldsymbol{M}}),
$$

with the inertial relaxation time $\eta$. In general, the Gilbert damping $\alpha$ and the inertial relaxation time $\eta$ are tensors [30], however, for an isotropic system, these parameters can be considered as scalars. The emergence of spin nutation has been attributed to an extension of the Kambersky breathing Fermi surface model [31,32], namely, an $s$ - $d$-like interaction spin model between local magnetization and itinerant electrons [33,34]. Moreover, the ILLG equation has been derived from the fundamental Dirac equation [20,30]. Note that the Gilbert damping and inertial relaxation time are related to each other as the Gilbert damping is associated with the imaginary part of the susceptibility, while the inertial dynamics are associated with the real part of the susceptibility $[20,35]$. The characteristic timescales of the nutation have been predicted to be in a range of $1-100$ fs $[26,33,36,37]$ and $1-10$ ps [37,38]. More recently, it has been demonstrated that simple classical mechanical considerations superimposed with Gilbert dynamics naturally lead to magnetic inertial dynamics $[39,40]$.

Theoretically, the spin nutation has recently been extensively discussed for one-sublattice ferromagnets $[26,37,41-$ 45]. The nutation resonance has also been observed in experiments, however, for two-sublattice ferromagnets [38]. A recent theoretical investigation predicts that the precession and nutation resonance frequencies may overlap in 
two-sublattice ferromagnets [46]. The spin-nutation resonance has been observed at a higher frequency than ferromagnetic resonance, e.g., while the ferromagnetic resonance occurs in the $\mathrm{GHz}$ regime, the nutation resonance occurs in the $\mathrm{THz}$ regime $[38,47]$. Moreover, the spin nutation shifts the ferromagnetic resonance frequency to a lower value. Although this shift is very small, the linewidth of the resonance decreases, however, and thus the effective damping decreases, too.

While the coupling of spin dynamics to mechanical inertia in antiferromagnets has been discussed [48], the pure spinnutation effects have not yet comprehensively been discussed in antiparallel aligned two-sublattice magnetic systems (e.g., antiferromagnets, ferrimagnets). In a recent investigation, it has been predicted that the spin nutation in antiferromagnets may have much significance [47]. Due to the sublattice exchange interaction, the antiferromagnetic resonance frequency lies in the $\mathrm{THz}$ regime, while the nutation resonance frequency has a similar order of magnitude. This helps to detect the antiferromagnetic precession and nutation resonances experimentally as they fall in the same frequency range. Moreover, the calculated shift of the antiferromagnetic resonance frequency is stronger than that of a ferromagnet. Additionally, the nutation resonance peak is exchange enhanced [47], which is beneficial for detection in experiments. However, the previous investigation only considers the intrasublattice inertial dynamics, while the effect of intersublattice inertial dynamics is unknown.

In a previous work, the LLG equation of motion with intersublattice Gilbert damping has been explored by Kamra et al. [49]. It was found that the introduction of intersublattice Gilbert damping enhances the damping [49-51]. In this paper, we formulate the spin dynamical equations in a two-sublattice magnetic system with both intra- and intersublattice inertial dynamics as well as inter- and intrasublattice Gilbert damping, thus extending previous work [47]. First, we derive the magnetic susceptibility with the intersublattice effects and compute the precession and nutation resonance frequencies. We find that the precession resonance frequency and the effective Gilbert damping decrease with the intrasublattice nutation coupling in antiferromagnets, however, they increase with the intersublattice nutation. Unlike antiferromagnets, we find for ferrimagnets that the change of precession resonance frequencies is more pronounced with both intra- and intersublattice nutation coupling constants in the exchangelike mode, but nearly negligible for the ferromagnetic mode.

The paper is organized as follows. First, in Sec. II, we discuss the linear-response theory of spin dynamics to calculate the magnetic susceptibility with the intra- and intersublattice nutation effects. In Sec. III, the precession resonance frequencies have been calculated with analytical and numerical tools for antiferromagnets (Sec. III A) and ferrimagnets (Sec. III B). We summarize the obtained results in Sec. IV.

\section{LINEAR-RESPONSE SUSCEPTIBILITY IN TWO-SUBLATTICE MAGNETS}

For two-sublattice magnetic systems, namely $A$ and $B$ representing the two sublattices, the ILLG equations of motion read

$$
\begin{aligned}
\dot{\boldsymbol{M}}_{A}= & -\gamma_{A}\left(\boldsymbol{M}_{A} \times \boldsymbol{H}_{A}\right)+\frac{\alpha_{A A}}{M_{A 0}}\left(\boldsymbol{M}_{A} \times \dot{\boldsymbol{M}}_{A}\right) \\
& +\frac{\alpha_{A B}}{M_{B 0}}\left(\boldsymbol{M}_{A} \times \dot{\boldsymbol{M}}_{B}\right)+\frac{\eta_{A A}}{M_{A 0}}\left(\boldsymbol{M}_{A} \times \ddot{\boldsymbol{M}}_{A}\right) \\
& +\frac{\eta_{A B}}{M_{B 0}}\left(\boldsymbol{M}_{A} \times \ddot{\boldsymbol{M}}_{B}\right), \\
\dot{\boldsymbol{M}}_{B}= & -\gamma_{B}\left(\boldsymbol{M}_{B} \times \boldsymbol{H}_{B}\right)+\frac{\alpha_{B B}}{M_{B 0}}\left(\boldsymbol{M}_{B} \times \dot{\boldsymbol{M}}_{B}\right) \\
& +\frac{\alpha_{B A}}{M_{A 0}}\left(\boldsymbol{M}_{B} \times \dot{\boldsymbol{M}}_{A}\right)+\frac{\eta_{B B}}{M_{B 0}}\left(\boldsymbol{M}_{B} \times \ddot{\boldsymbol{M}}_{B}\right) \\
& +\frac{\eta_{B A}}{M_{A 0}}\left(\boldsymbol{M}_{B} \times \ddot{\boldsymbol{M}}_{A}\right) .
\end{aligned}
$$

In the above dynamical equations, the first terms relate to the spin precession, the second and third terms represent the intraand intersublattice Gilbert damping, and the last two terms classify the intra- and intersublattice inertial dynamics. The intrasublattice magnetization dynamics has been characterized with the Gilbert damping constants $\alpha_{A A}, \alpha_{B B}$ and inertial relaxation time $\eta_{A A}$ or $\eta_{B B}$, while the intersublattice dynamics is characterized by Gilbert damping $\alpha_{A B}$ or $\alpha_{B A}$ and inertial relaxation time $\eta_{A B}$ or $\eta_{B A}$. Note that the Gilbert damping parameters are dimensionless, however, inertial relaxation time has a dimension of time $[26,27,30]$. The extended equations of motions in Eqs. (3) and (4) represent general magnetization dynamics for two-sublattice magnets (e.g., antiferromagnets, ferrimagnets, two-sublattice ferromagnets, and so on).

The free energy of the considered two-sublattice system reads

$$
\begin{aligned}
\mathcal{F}\left(\boldsymbol{M}_{A}, \boldsymbol{M}_{B}\right)= & -H_{0}\left(M_{A z}+M_{B z}\right)-\frac{K_{A}}{M_{A 0}^{2}} M_{A z}^{2} \\
& -\frac{K_{B}}{M_{B 0}^{2}} M_{B z}^{2}+\frac{J}{M_{A 0} M_{B 0}} \boldsymbol{M}_{A} \cdot \boldsymbol{M}_{B} .
\end{aligned}
$$

Here, the first term defines the Zeeman coupling of two sublattice spins with an external field $\boldsymbol{H}_{0}=H_{0} \hat{z}$. The second and third terms represent the anisotropy energies for the sublattice $A$ and $B$, respectively. The last term can be identified as the Heisenberg exchange energy between the two sublattices. Note that the Heisenberg coupling energy $J>0$ for antiferromagnets and ferrimagnets, but $J<0$ for ferromagneticlike coupling.

We calculate the effective field in the ILLG equation as the derivative of free energy in Eq. (5) with respect to the corresponding magnetization

$$
\begin{aligned}
\boldsymbol{H}_{A} & =-\frac{\partial \mathcal{F}\left(\boldsymbol{M}_{A}, \boldsymbol{M}_{B}\right)}{\partial \boldsymbol{M}_{A}} \\
& =\left(H_{0}+\frac{2 K_{A}}{M_{A 0}^{2}} M_{A z}\right) \hat{z}-\frac{J}{M_{A 0} M_{B 0}} \boldsymbol{M}_{B}, \\
\boldsymbol{H}_{B} & =-\frac{\partial \mathcal{F}\left(\boldsymbol{M}_{A}, \boldsymbol{M}_{B}\right)}{\partial \boldsymbol{M}_{B}} \\
& =\left(H_{0}+\frac{2 K_{B}}{M_{B 0}^{2}} M_{B z}\right) \hat{z}-\frac{J}{M_{A 0} M_{B 0}} \boldsymbol{M}_{A} .
\end{aligned}
$$

First, in the ground state, we consider that the $A$ sublattice magnetization is $\boldsymbol{M}_{A}=M_{A 0} \hat{z}$, while the $B$ sublattice 
magnetization is antiparallel $\boldsymbol{M}_{B}=-M_{B 0} \hat{\boldsymbol{z}}$, such that we can describe the antiferromagnets $\left(M_{A 0}=M_{B 0}\right)$ and ferrimagnets $\left(M_{A 0}>M_{B 0}\right)$. We then expand the magnetization around the ground state in small deviations, $\boldsymbol{M}_{A}=M_{A 0} \hat{z}+\boldsymbol{m}_{A}(t)$ and $\boldsymbol{M}_{B}=-M_{B 0} \hat{z}+\boldsymbol{m}_{B}(t)$. The small deviations $\boldsymbol{m}_{A / B}$ are induced by the transverse external field $\boldsymbol{h}_{A / B}(t)$.

For convenience, we work in the circularly polarized basis, i.e., $m_{A / B \pm}=m_{A / B x} \pm i m_{A / B y}, \quad h_{A / B \pm}=h_{A / B x} \pm i h_{A / B y}$, and define $\Omega_{A}=\gamma_{A} / M_{A 0}\left(J+2 K_{A}+H_{0} M_{A 0}\right), \Omega_{B}=\gamma_{B} / M_{B 0}(J+$ $\left.2 K_{B}-H_{0} M_{B 0}\right)$. With the time-dependent harmonic fields and magnetizations $h_{A / B \pm}, m_{A / B \pm} \propto e^{ \pm i \omega t}$, we obtain the magnetic susceptibility tensor [47]

$$
\begin{aligned}
\left(\begin{array}{l}
m_{A \pm} \\
m_{B \pm}
\end{array}\right) & =\frac{1}{\Delta_{ \pm}}\left(\begin{array}{l}
\frac{1}{\gamma_{B} M_{B 0}}\left(\Omega_{B} \pm i \omega \alpha_{B B}-\omega^{2} \eta_{B B}+\omega\right) \\
-\frac{1}{\gamma_{A} M_{B 0}}\left(\frac{\gamma_{A}}{M_{A 0}} J \pm i \omega \alpha_{A B}-\omega^{2} \eta_{A B}\right)
\end{array}\right. \\
& =\chi_{ \pm}^{A B}\left(\begin{array}{l}
h_{A \pm} \\
h_{B \pm}
\end{array}\right)
\end{aligned}
$$

with the definition of the determinant $\Delta_{ \pm}=$ $\left(\gamma_{A} \gamma_{B} M_{A 0} M_{B 0}\right)^{-1}\left(\Omega_{A} \pm i \omega \alpha_{A A}-\omega^{2} \eta_{A A}-\omega\right)\left(\Omega_{B} \pm\right.$ $\left.i \omega \alpha_{B B}-\omega^{2} \eta_{B B}+\omega\right)-\left(\gamma_{A} \gamma_{B} M_{A 0} M_{B 0}\right)^{-1}\left(\frac{\gamma_{A}}{M_{A 0}} J \pm i \omega \alpha_{A B}-\right.$ $\left.\omega^{2} \eta_{A B}\right)\left(\frac{\gamma_{B}}{M_{B 0}} J \pm i \omega \alpha_{B A}-\omega^{2} \eta_{B A}\right)$.

As one expects, the intersublattice Gilbert damping and inertial dynamical contributions arise in the off-diagonal components of the susceptibility tensor, while the intrasublattice contributions are in the diagonal component of the susceptibility [47]. Note that without inertial dynamics terms, the expression for the susceptibility is in accordance with the one derived by Kamra et al. [49].

To find the resonance frequencies, the determinant $\Delta_{ \pm}$ must go to zero, thus one has to solve the following fourthorder equation in frequency

$$
\mathcal{A}_{ \pm} \omega^{4}+\mathcal{B}_{ \pm} \omega^{3}+\mathcal{C}_{ \pm} \omega^{2}+\mathcal{D}_{ \pm} \omega+\mathcal{E}_{ \pm}=0
$$

where the coefficients have the following forms,

$$
\begin{aligned}
\mathcal{A}_{ \pm}= & \eta_{A A} \eta_{B B}-\eta_{A B} \eta_{B A}, \\
\mathcal{B}_{ \pm}= & \mp i\left(\alpha_{A A} \eta_{B B}+\alpha_{B B} \eta_{A A}\right)-\left(\eta_{A A}-\eta_{B B}\right) \\
& \pm i\left(\alpha_{A B} \eta_{B A}+\alpha_{B A} \eta_{A B}\right) \\
\mathcal{C}_{ \pm}= & -1 \pm i\left(\alpha_{A A}-\alpha_{B B}\right)-\left(\Omega_{A} \eta_{B B}+\Omega_{B} \eta_{A A}\right) \\
& -\alpha_{A A} \alpha_{B B}+\left(\frac{\gamma_{A}}{M_{A 0}} \eta_{B A}+\frac{\gamma_{B}}{M_{B 0}} \eta_{A B}\right) J \\
& +\alpha_{A B} \alpha_{B A}, \\
\mathcal{D}_{ \pm}= & \left(\Omega_{A}-\Omega_{B}\right) \pm i\left(\Omega_{A} \alpha_{B B}+\Omega_{B} \alpha_{A A}\right) \\
& \times \mp i\left(\frac{\gamma_{A}}{M_{A 0}} \alpha_{B A}+\frac{\gamma_{B}}{M_{B 0}} \alpha_{A B}\right) J \\
\mathcal{E}_{ \pm}= & \Omega_{A} \Omega_{B}-\frac{\gamma_{A} \gamma_{B}}{M_{A 0} M_{B 0}} J^{2} .
\end{aligned}
$$

The solutions of the above equation (9) result in four different frequencies in the presence of a finite external field. Two of those frequencies can be associated with the magnetization precession resonance $\omega_{\mathrm{p} \pm}$ (positive and negative modes) that exists even without nutation. The other two frequencies dictate the nutation resonance frequencies $\omega_{\mathrm{n} \pm}$ (positive and negative modes).

\section{RESULTS AND DISCUSSION}

The intrinsic intrasublattice inertial dynamics have been discussed extensively in Ref. [47]. Essentially, the resonance frequencies and effective damping decrease with increasing intrasublattice inertial relaxation time for antiferromagnets and ferrimagnets. Therefore, we consider a constant intrasublattice inertial relaxation time in this work. In this section, we specifically discuss the effects of intersublattice nutation in both antiferromagnets and ferrimagnets.

\section{A. Antiferromagnets}

To start with, we calculate the frequency-dependent dissipated power of an antiferromagnet. Using the expressions for the susceptibility in Eq. (8), we calculate the dissipated power in the inertial dynamics with the following definition, $P_{A B}=\dot{\boldsymbol{m}}_{A} \cdot \boldsymbol{h}_{A}+\dot{\boldsymbol{m}}_{B} \cdot \boldsymbol{h}_{B}=\frac{1}{2}\left(\dot{m}_{A+} h_{A-}+\dot{m}_{A-} h_{A+}+\right.$ $\dot{m}_{B+} h_{B-}+\dot{m}_{B-} h_{B+}$ ), which leads to a complicated expression (not given). For convenience, we define $\alpha_{A A}=\alpha_{B B}=$ $\alpha, \eta_{A A}=\eta_{B B}=\eta$. To focus on the intersublattice nutation $\eta_{A B}=\eta_{B A}=\eta^{\prime}$, we set the intersublattice Gilbert damping to zero, i.e., $\alpha_{A B}=\alpha_{B A}=0$, and choose $M_{A 0}=M_{B 0}=2 \mu_{B}$. The exchange and anisotropy energies, magnetic moments used in the here-presented computations are comparable to a typical antiferromagnetic $\mathrm{NiO}[23,52,53]$ or $\mathrm{CoO}[54,55]$ system. However, we mention that $\mathrm{NiO}$ or $\mathrm{CoO}$ bulk crystals have biaxial anisotropy. Also, the Gilbert damping of $\mathrm{NiO}$ is very small, $\alpha_{A A}=\alpha_{B B}=\alpha \sim 10^{-4}$, i.e., less than the here-used value. In contrast, a large spin-orbit coupling in antiferromagnetic $\mathrm{CrPt}$ (that has $\sim 2 \mu_{B} \mathrm{Cr}$ moments) leads to a higher Gilbert damping $\alpha \sim 10^{-2}$ [56,57]. Importantly, the inertial relaxation times $\eta$ and $\eta^{\prime}$ are not known in these antiferromagnetic systems. Our simulations pertain therefore to typical, selected model systems. We show the evaluated dissipated power with and without inertial dynamics for such an antiferromagnet in Fig. 1. Note that the dissipated power has already been calculated in Ref. [47], however, without the intersublattice inertial dynamics. We can observe that while the intrasublattice inertial dynamics decreases the precessional resonance frequencies (see the cyan lines in Fig. 1), the intersublattice inertial dynamics works oppositely. Note that the nutation resonance frequencies decrease with the introduction of intersublattice inertial dynamics.

To understand the effect of the intersublattice nutation terms, first, we solve Eq. (9), considering again $\alpha_{A A}=$ $\alpha_{B B}=\alpha, \eta_{A A}=\eta_{B B}=\eta, \eta_{A B}=\eta_{B A}=\eta^{\prime}$, and $\alpha_{A B}=\alpha_{B A}=$ 0 . As the nutation in antiferromagnets is exchange enhanced [47], we calculate the effect of intersublattice terms 


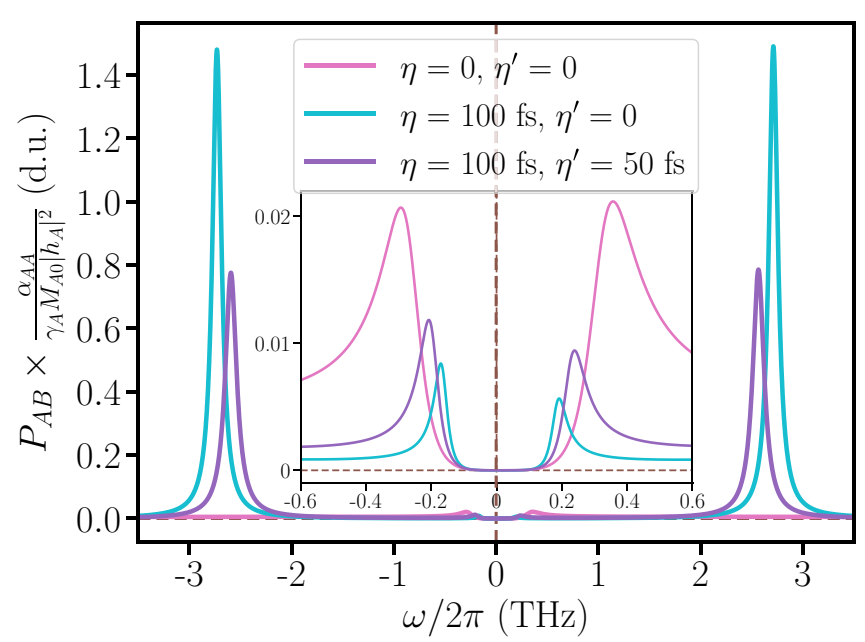

FIG. 1. The calculated dissipated power vs frequency for an antiferromagnet with $M_{A 0}=M_{B 0}=2 \mu_{B}$, and various values of the intra- and intersublattice nutation parameters $\eta$ and $\eta^{\prime}$. The inset shows the dissipated power in dimensionless units (d.u.) close to the precession resonance frequencies. The other used parameters are $\gamma_{A}=\gamma_{B}=1.76 \times 10^{11} \mathrm{~T}^{-1} \mathrm{~s}^{-1}, J=10^{-21} \mathrm{~J}, K_{A}=K_{B}=10^{-23} \mathrm{~J}$, $H_{0}=1 \mathrm{~T}, \alpha_{A A}=\alpha_{B B}=0.05, \alpha_{A B}=\alpha_{B A}=0, \eta_{A A}=\eta_{B B}=\eta$, and $\eta_{A B}=\eta_{B A}=\eta^{\prime}$.

on the precession and nutation frequencies, setting $\gamma_{A}=$ $\gamma_{B}=\gamma$ and $M_{A 0}=M_{B 0}=M_{0}$ for antiferromagnets. Therefore, the fourth-order equation in Eq. (9) reduces to an equation with $\mathcal{A}_{ \pm}^{\mathrm{AFM}}=\eta^{2}-\eta^{\prime 2}, \mathcal{B}_{ \pm}^{\mathrm{AFM}}=\mp i 2 \alpha \eta, \mathcal{C}_{ \pm}^{\mathrm{AFM}}=$ $-1-\left(\Omega_{A}+\Omega_{B}\right) \eta+2 \frac{\gamma}{M_{0}} \eta^{\prime} J, \mathcal{D}_{ \pm}^{\mathrm{AFM}}=\left(\Omega_{A}-\Omega_{B}\right) \pm i\left(\Omega_{A}+\right.$ $\left.\Omega_{B}\right) \alpha$, and $\mathcal{E}_{ \pm}^{\mathrm{AFM}}=\Omega_{A} \Omega_{B}-\left(\frac{\gamma}{M_{0}} J\right)^{2}$. The solution of the above equation results in precession and nutation resonance frequencies for the two modes (positive and negative). Inserting the real and imaginary parts of the solutions $\omega_{ \pm}=$ $\operatorname{Re}\left(\omega_{ \pm}\right)+i \operatorname{Im}\left(\omega_{ \pm}\right)$, we numerically calculate the precession resonance frequencies and effective damping (the ratio of imaginary and real frequencies) for an antiferromagnet as a function of intersublattice nutation. The results are shown in
Fig. 2, where the data points correspond to the numerical solutions.

On the other hand, the fourth-order equation, $\mathcal{A}_{+}^{\mathrm{AFM}} \omega^{4}+$ $\mathcal{B}_{+}^{\mathrm{AFM}} \omega^{3}+\mathcal{C}_{+}^{\mathrm{AFM}} \omega^{2}+\mathcal{D}_{+}^{\mathrm{AFM}} \omega+\mathcal{E}_{+}^{\mathrm{AFM}}=0$, can analytically be solved using the considerations that $K_{A}=K_{B}=K, J \gg K$, $M_{0} H_{0}$, and $\alpha \ll 1$. Therefore, one has $\Omega_{A}=\Omega_{B} \approx \gamma(J+$ $2 K) / M_{0}$. Essentially, the fourth-order equation reduces to

$$
\begin{gathered}
\left(\eta^{2}-\eta^{\prime 2}\right) \omega^{4}-\left[1+2 \frac{\gamma \eta(J+2 K)}{M_{0}}-2 \frac{\gamma \eta^{\prime} J}{M_{0}}\right] \omega^{2} \\
-2 i \alpha \eta \omega_{(0)}^{3}+2 \gamma H_{0} \omega_{(0)}+\frac{2 i \gamma \alpha}{M_{0}}(J+2 K) \omega_{(0)} \\
+\frac{\gamma^{2}}{M_{0}^{2}}(J+2 K)^{2}-\frac{\gamma^{2} J^{2}}{M_{0}^{2}}-\gamma^{2} H_{0}^{2}=0
\end{gathered}
$$

with $\omega_{(0)}$ being the solution of the above equation for $\alpha=0$ and $H_{0}=0$. The solutions of the above equation are rather simple and provide the two precession frequency modes (positive and negative) for antiferromagnets. Expanding the solutions of Eq. (15) up to the first order in $\alpha$ and $H_{0}$, and also in first order in $K / J \ll 1$, the precession resonance frequencies are obtained (neglecting the higher-order in $\omega_{(0)}$ terms) as

$$
\begin{aligned}
\omega_{\mathrm{p} \pm} \approx & \pm \frac{\gamma}{M_{0}} \frac{\sqrt{4 K(K+J)}}{\sqrt{1+\frac{4 \gamma \eta K}{M_{0}}+\frac{2 \gamma J}{M_{0}}\left(\eta-\eta^{\prime}\right)}} \\
& +\frac{\gamma H_{0}+i \frac{\gamma \alpha}{M_{0}}(J+2 K)}{\sqrt{1+\frac{4 \gamma \eta K}{M_{0}}+\frac{2 \gamma J}{M_{0}}\left(\eta-\eta^{\prime}\right)}} \frac{\left|\omega_{(0)}\right|}{\frac{\gamma}{M_{0}} \sqrt{4 K(K+J)}}
\end{aligned}
$$

Now substituting the $\left|\omega_{(0)}\right|$ from the leading term in the frequency expression into the perturbative terms in Eq. (16), the approximate precession frequencies are
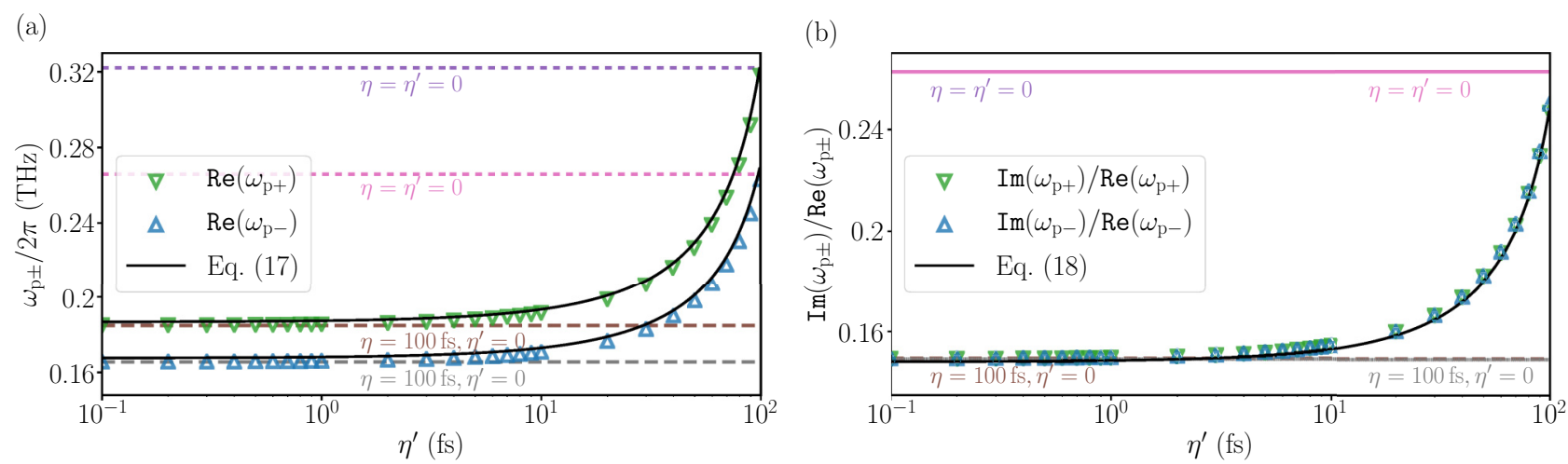

FIG. 2. The calculated precession frequencies as a function of intersublattice nutation $\eta^{\prime}$ for an antiferromagnet, setting $M_{A 0}=M_{B 0}=2 \mu_{B}$. The data points denote the numerical solution of Eq. (9) and the black lines correspond to the analytical solution in Eq. (16). (a) The real part of the resonance frequency, and (b) the ratio of imaginary and real part of the frequency have been plotted. The other used parameters are $\gamma_{A}=$ $\gamma_{B}=1.76 \times 10^{11} \mathrm{~T}^{-1} \mathrm{~s}^{-1}, J=10^{-21} \mathrm{~J}, K_{A}=K_{B}=K=10^{-23} \mathrm{~J}, H_{0}=1 \mathrm{~T}, \alpha_{A A}=\alpha_{B B}=\alpha=0.05, \alpha_{A B}=\alpha_{B A}=0, \eta_{A A}=\eta_{B B}=\eta=100$ fs, and $\eta_{A B}=\eta_{B A}=\eta^{\prime}$. The horizontal lines correspond to solutions with zero intersublattice nutation $\left(\eta^{\prime}=0\right)$. Note that we show Re $\left(\omega_{\mathrm{p}-}\right)$ as $-\operatorname{Re}\left(\omega_{\mathrm{p}-}\right)$. 
obtained as

$$
\begin{aligned}
\omega_{\mathrm{p} \pm} \approx & \pm \frac{\gamma}{M_{0}} \frac{\sqrt{4 K(K+J)}}{\sqrt{1+\frac{4 \gamma \eta K}{M_{0}}+\frac{2 \gamma J}{M_{0}}\left(\eta-\eta^{\prime}\right)}} \\
& +\frac{\gamma H_{0}+i \frac{\gamma \alpha}{M_{0}}(J+2 K)}{1+\frac{4 \gamma \eta K}{M_{0}}+\frac{2 \gamma J}{M_{0}}\left(\eta-\eta^{\prime}\right)} .
\end{aligned}
$$

This equation has been plotted in Fig. 2 as black lines. Note that for $\omega_{\mathrm{p}-}$ we show for convenience $-\operatorname{Re}\left(\omega_{\mathrm{p}-}\right)$ in Fig. 2(a) and in the following. Due to the presence of $\eta-\eta^{\prime}$ in the denominator of the frequency expressions, the precession resonance frequency increases when intersublattice nutation is taken into account $\left(\eta^{\prime}<\eta\right)$, which explains the increase in frequency in Fig. 2(a). At the limit $\eta \rightarrow \eta^{\prime}$, the nutation (intra- and intersublattice) does not play a significant role as the precession resonance frequency is decreased by a factor $\sqrt{1+\frac{4 \gamma \eta K}{M_{0}}}$ which is very small due to $K \ll J$. We emphasize that the overall inertial relaxation time of a physical system has to be positive definite, which means that $\eta^{\prime}$ is bounded, $\eta^{\prime} \leqslant \eta$. The values of $\eta^{\prime}>\eta$ provide an unphysical result. Note that the two resonance frequencies are approximately 0.332 and $0.276 \mathrm{THz}$ with $\alpha=0$ and $\eta=\eta^{\prime}=0$, while these two frequencies are 0.322 and $0.266 \mathrm{THz}$ with $\alpha=0.05$ and $\eta=\eta^{\prime}=0$. The latter has been shown in Fig. 2(a) as dashed lines. Therefore, the Gilbert damping has already the effect that it reduces the resonance frequencies.

The effective Gilbert damping can be calculated using the ratio between the imaginary and real parts of the frequencies, i.e., the linewidth. From Eq. (17) one arrives at

$$
\frac{\operatorname{Im}\left(\omega_{\mathrm{p} \pm}\right)}{\operatorname{Re}\left(\omega_{\mathrm{p} \pm}\right)} \approx \alpha \frac{(J+2 K)}{\sqrt{4 K(K+J)}} \frac{1}{\sqrt{1+\frac{4 \gamma \eta K}{M_{0}}+\frac{2 \gamma J}{M_{0}}\left(\eta-\eta^{\prime}\right)}} .
$$

Note that the two resonance modes have the same effective damping. For ferromagnets, the exchange energies do not contribute and thus the effective damping remains the same as $\alpha$, in the absence of magnetic inertial terms (see Ref. [46]). However, in antiferromagnets the effective damping is enhanced due to the exchange interaction by a factor $\frac{(J+2 K)}{\sqrt{4 K(K+J)}}$, even without any inertial terms. As investigated earlier [47], the effective damping decreases with the intrasublattice relaxation time. However, similar to the increase in frequency, the effective damping also increases with the intersublattice inertial relaxation time, as seen in Fig. 2(b). The analytical solution in Eq. (18) agrees excellently with the numerical solutions. Close to the limit $\eta^{\prime} \rightarrow \eta$, the effective Gilbert damping in Eq. (18), one expects the effective damping to be increased by a factor $\left(1+\frac{4 \gamma \eta K}{M_{0}}\right)^{-1 / 2}$, as can be seen in Fig. 2(b).

Next, we discuss the field dependence of the resonance frequencies. The precession resonance frequencies and effective damping have been plotted as a function of the applied field $H_{0}$ for several intersublattice relaxation times in Fig. 3. As can be observed, at zero applied field, the two modes (positive and negative) coincide in antiferromagnets, a fact that can be seen from Eq. (17). However, the applied field induces the splitting of these two modes. The frequency splitting
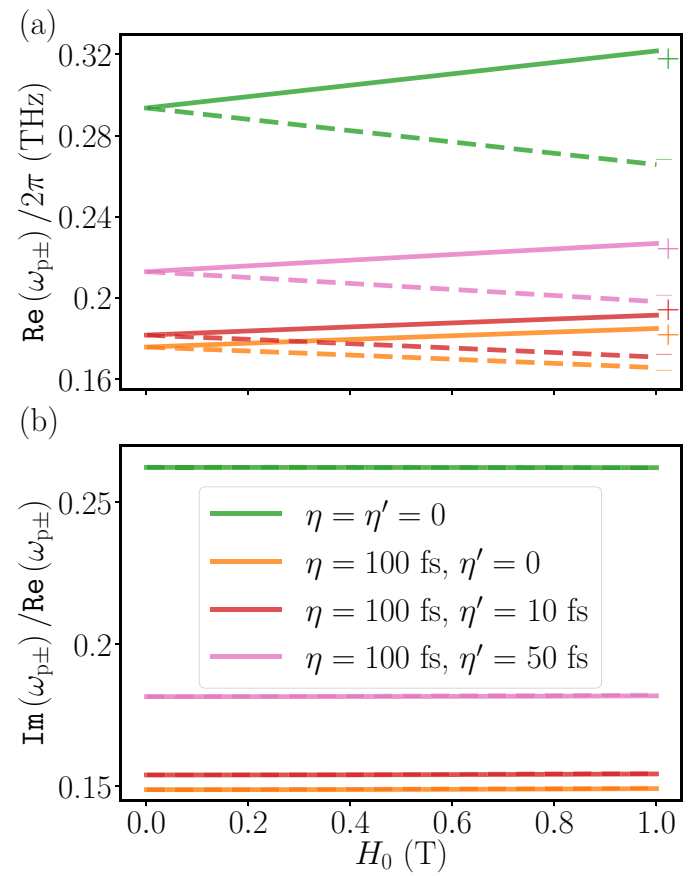

FIG. 3. The calculated precession frequencies at several intersublattice relaxation times as a function of applied field for antiferromagnets using $M_{A 0}=M_{B 0}=2 \mu_{B}$. The solid and dashed lines represent the positive and negative modes, respectively. (a) The real part of the resonance frequencies and (b) the ratio of imaginary and real part of the frequency have been plotted. The other used parameters are $\gamma_{A}=\gamma_{B}=1.76 \times 10^{11} \mathrm{~T}^{-1} \mathrm{~s}^{-1}, J=10^{-21} \mathrm{~J}, K_{A}=$ $K_{B}=K=10^{-23} \mathrm{~J}, \alpha_{A A}=\alpha_{B B}=\alpha=0.05, \alpha_{A B}=\alpha_{B A}=0, \eta_{A A}=$ $\eta_{B B}=\eta=100 \mathrm{fs}$, and $\eta_{A B}=\eta_{B A}=\eta^{\prime}$.

scales with $\left[1+\frac{4 \gamma \eta K}{M_{0}}+\frac{2 \gamma J}{M_{0}}\left(\eta-\eta^{\prime}\right)\right]^{-1} \gamma H_{0}$, meaning that the splitting is linear in the applied field $H_{0}$. On the other hand, at a constant field, the splitting also depends on the interand intrasublattice nutation. From Eq. (17), it is clear that the splitting is reduced with intrasublattice nutation, while it is enhanced with intersublattice nutation. Such a conclusion can also be drawn from the numerical solutions in Fig. 3(a). The effective damping of the antiferromagnet remains field independent which can be observed in Fig. 3(b).

Proceeding as previously, we obtain the following nutation frequencies,

$$
\begin{aligned}
\omega_{\mathrm{n} \pm} \approx & \pm \frac{1}{\eta} \sqrt{\frac{1+\frac{4 \gamma \eta K}{M_{0}}+\frac{2 \gamma J}{M_{0}}\left(\eta-\eta^{\prime}\right)}{1-\frac{\eta^{2}}{\eta^{2}}}} \\
& \times\left(1-\frac{\frac{4 \gamma^{2} K(J+K)\left(\eta^{2}-\eta^{\prime 2}\right)}{M_{0}}}{2\left[1+\frac{4 \gamma \eta K}{M_{0}}+\frac{2 \gamma J}{M_{0}}\left(\eta-\eta^{\prime}\right)\right]^{2}}\right) \\
& -\frac{\gamma H_{0}-i \alpha\left[\frac{\eta}{\eta^{2}-\eta^{2}}+\frac{\gamma}{M_{0}}(J+2 K)\right]}{1+\frac{4 \gamma \eta K}{M_{0}}+\frac{2 \gamma J}{M_{0}}\left(\eta-\eta^{\prime}\right)} .
\end{aligned}
$$

Note that at the limit $\eta^{\prime} \rightarrow 0$, the nutation frequencies without the intersublattice coupling are recovered [47]. The dominant term in the calculated frequency is the first term in Eq. (19). With the introduction of intersublattice coupling 
(a)

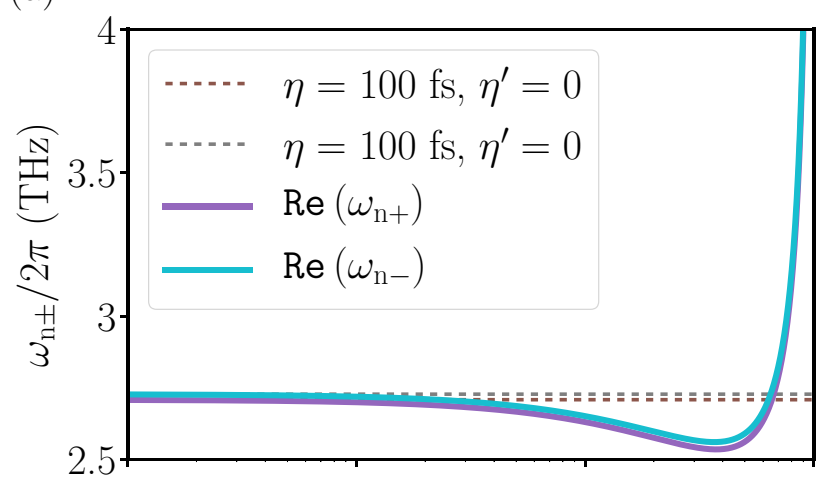

(b)

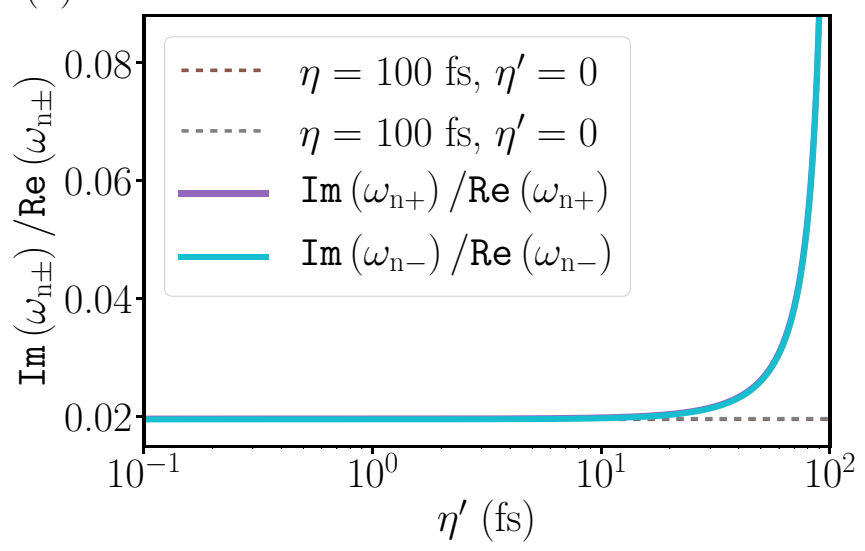

FIG. 4. The calculated nutation frequencies as a function of intersublattice nutation for antiferromagnets using $M_{A 0}=M_{B 0}=2 \mu_{B}$. (a) The real part of the nutation resonance frequencies and (b) the ratio of imaginary and real part of the nutation resonance frequency have been plotted. The other used parameters are $\gamma_{A}=\gamma_{B}=$ $1.76 \times 10^{11} \mathrm{~T}^{-1} \mathrm{~s}^{-1}, J=10^{-21} \mathrm{~J}, K_{A}=K_{B}=K=10^{-23} \mathrm{~J}, H_{0}=$ $1 \mathrm{~T}, \alpha_{A A}=\alpha_{B B}=\alpha=0.05, \quad \alpha_{A B}=\alpha_{B A}=0, \quad \eta_{A A}=\eta_{B B}=\eta=$ $100 \mathrm{fs}$, and $\eta_{A B}=\eta_{B A}=\eta^{\prime}$.

$\eta^{\prime}$, both the numerator and denominator of the dominant frequency term decrease and therefore the nutation frequencies approximately stay constant (with a slow decrease) with intersublattice nutation when $\eta>\eta^{\prime}$ as plotted in Fig. 4. However, in the limit $\eta^{\prime} \rightarrow \eta$, the denominator vanishes, and thus the nutation frequencies diverge as can be seen in Fig. 4. It is interesting to note that the intersublattice inertial dynamics increase the precession resonance frequencies, but decrease the nutation frequency. Such an observation is also consistent with the dissipated power in Fig. 1. The damping of the inertial dynamics also shows a similar behavior: It stays nearly constant with a divergence at the limit $\eta^{\prime} \rightarrow \eta$.

As mentioned before, the inertial relaxation times $\eta$ and $\eta^{\prime}$ are not known for typical antiferromagnetic systems. Notwithstanding, we obtain the general result that the precession resonance frequencies decrease with intrasublattice inertial dynamics, but increase with intersublattice inertial dynamics. Thus, to experimentally realize the signature of inertial dynamics, an antiferromagnet with a higher ratio of intrato intersublattice inertial relaxation time $\left(\eta / \eta^{\prime} \gg 1\right)$ is better suited.
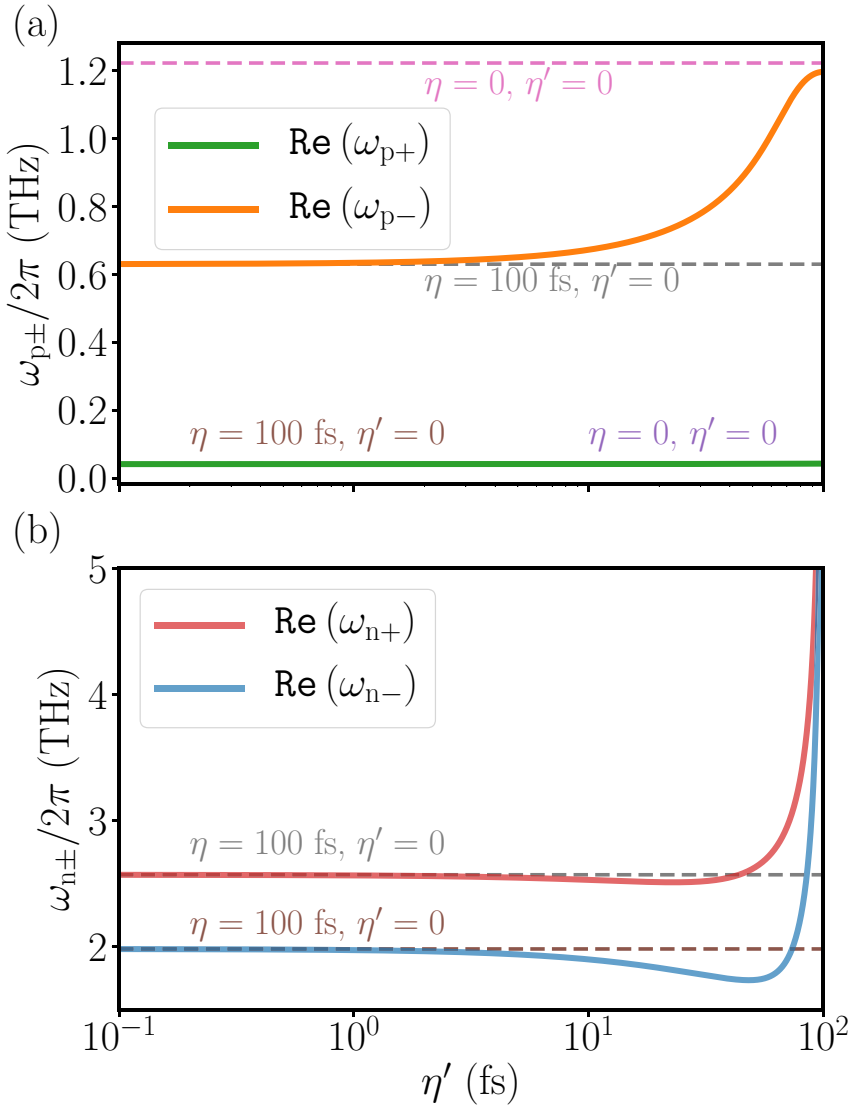

FIG. 5. The calculated precession and nutation frequencies as a function of intersublattice nutation for ferrimagnets using $M_{A 0}=$ $5 M_{B 0}=10 \mu_{B}$. The real part of the (a) precession resonance and (b) nutation resonance frequencies have been plotted. The other used parameters are $\gamma_{A}=\gamma_{B}=1.76 \times 10^{11} \mathrm{~T}^{-1} \mathrm{~s}^{-1}, J=10^{-21} \mathrm{~J}$, $K_{A}=K_{B}=K=10^{-23} \mathrm{~J}, H_{0}=1 \mathrm{~T}, \alpha_{A A}=\alpha_{B B}=\alpha=0.05, \alpha_{A B}=$ $\alpha_{B A}=0, \eta_{A A}=\eta_{B B}=\eta=100 \mathrm{fs}$, and $\eta_{A B}=\eta_{B A}=\eta^{\prime}$.

\section{B. Ferrimagnets}

Next, we consider a ferrimagnetic system where the magnetic moments in the two sublattices are different, i.e., $M_{A 0} \neq$ $M_{B 0}$. In this case, the analytical solution of Eq. (9) becomes cumbersome. The main reason is that $\Omega_{A} \neq \Omega_{B}$ for ferrimagnets, in fact, we calculate $\Omega_{A}-\Omega_{B}=\frac{\gamma(J+2 K)\left(M_{A 0}-M_{B 0}\right)}{M_{A 0} M_{B 0}}+$ $2 \gamma H_{0}$. For antiferromagnets, the magnetic moments in the two sublattices are exactly the same, i.e., $M_{A 0}=M_{B 0}$, and thus, within the approximation of $J \gg M_{0} H_{0}$, we find $\Omega_{A}=\Omega_{B}$ which simplifies the analytical solution of Eq. (9). Thus, we numerically solve Eq. (9) to calculate the precession and nutation resonance frequencies for ferrimagnets. We consider the case where $M_{A 0}=10 \mu_{B}$ and $M_{B 0}=2 \mu_{B}$, reminiscent of rare-earth-transition-metal ferrimagnets such as $\mathrm{GdFeCo}$ [2,3] or TbCo [58-60]. However, we emphasize that the inertial relaxation times $\eta$ and $\eta^{\prime}$ are not known for these materials. The calculated precession frequencies are shown in Fig. 5(a). The effect of intrasublattice inertial dynamics has already been studied in Ref. [47]. For ferrimagnets, the negative frequency mode appears to have a higher frequency (i.e., larger negative) than the positive one. However, both precession frequencies decrease with intrasublattice relaxation 
time $\eta$ [47]. We therefore have set the intrasublattice relaxation time $\eta$ to $100 \mathrm{fs}$ and vary the intersublattice relaxation time $\eta^{\prime}<\eta$. The upper precession resonance mode $\omega_{\mathrm{p}-}-$ the exchangelike mode-increases with the intersublattice relaxation time $\eta^{\prime}$, while the ferromagneticlike mode $\omega_{\mathrm{p}+}$ shows a very small increase. Thus, for ferrimagnets, the change in precession frequencies is more significant in the exchangelike mode than in the ferromagneticlike mode. At the limit $\eta^{\prime} \rightarrow \eta$, the precession resonance frequencies almost coincide with the resonance frequencies calculated at $\eta=\eta^{\prime}=0$, meaning that the inertial dynamics do not play any role for the precession resonance frequency. The latter can clearly be seen in Fig. 5(a). These observations are similar to the antiferromagnet as discussed earlier. The nutation resonance frequencies in Fig. 5(b) again decline with the intersublattice relaxation time showing a divergence at the limit $\eta^{\prime} \rightarrow \eta$. However, one can notice here two distinguishable nutation resonance frequencies unlike almost the single-valued nutation frequencies of antiferromagnets.

\section{SUMMARY}

In summary, we have formulated a linear-response theory of the ILLG equations for antiferromagnets with inter- and intrasublattice inertial dynamics. The calculation of the susceptibility tensor shows that the intrasublattice terms appear in the diagonal elements, while the intersublattice terms appear in the off-diagonal elements. The dissipated power contains a precession resonance peak in the sub- $\mathrm{THz}$ regime for antiferromagnets, however, the introduction of inertial dynamics causes another peak, a nutation resonance peak at a higher, few $\mathrm{THz}$ frequency. Moreover, we observe that the intersublattice inertial dynamics work oppositely to the intrasublattice inertial one. By finding the poles of the susceptibility, we calculate the precession and nutation resonance frequencies. While the precession resonance frequencies decrease with intrasublattice relaxation time, the intersublattice inertial dynamics have the opposite effect. In fact, we observe that the magnetic inertia does not have any effect on the antiferromagnetic precession resonance at the limit $\eta^{\prime} \rightarrow \eta$. On the other hand, the $\mathrm{THz}$ nutation resonance frequency decreases slightly with the introduction of intersublattice inertial dynamics, however, showing a divergence at the limit $\eta^{\prime} \rightarrow \eta$. Our derived analytical theory explains such intersublattice contributions. Finally, for ferrimagnets, we find a similar behavior for the intersublattice inertial dynamics. However, the precession resonance frequency of the exchangelike mode depends significantly on the nutation couplings in contrast to that of the ferromagneticlike mode that is practically independent of the nutation constants.

\section{ACKNOWLEDGMENTS}

We acknowledge Levente Rózsa and Ulrich Nowak for fruitful discussions, the Swedish Research Council (VR Grant No. 2019-06313) for research funding, and Swedish National Infrastructure for Computing (SNIC) at NSC Linköping for computational resources. We further acknowledge support through the European Union's Horizon 2020 Research and Innovation Programme under Grant Agreement No. 863155 (s-Nebula).
[1] E. Beaurepaire, J.-C. Merle, A. Daunois, and J.-Y. Bigot, Phys. Rev. Lett. 76, 4250 (1996).

[2] C. D. Stanciu, F. Hansteen, A. V. Kimel, A. Kirilyuk, A. Tsukamoto, A. Itoh, and T. Rasing, Phys. Rev. Lett. 99, 047601 (2007).

[3] K. Vahaplar, A. M. Kalashnikova, A. V. Kimel, D. Hinzke, U. Nowak, R. Chantrell, A. Tsukamoto, A. Itoh, A. Kirilyuk, and T. Rasing, Phys. Rev. Lett. 103, 117201 (2009).

[4] K. Carva, P. Baláž, and I. Radu, Laser-induced ultrafast magnetic phenomena, in Handbook of Magnetic Materials, edited by E. Brück (Elsevier, Amsterdam, 2017), Vol. 26, pp. 291-463.

[5] R. John, M. Berritta, D. Hinzke, C. Müller, T. Santos, H. Ulrichs, P. Nieves, J. Walowski, R. Mondal, O. ChubykaloFesenko, J. McCord, P. M. Oppeneer, U. Nowak, and M. Münzenberg, Sci. Rep. 7, 4114 (2017).

[6] L. D. Landau and E. M. Lifshitz, Ukr. J. Phys. 53, 14 (2008) [Phys. Z. Sowjetunion 8, 153 (1935)].

[7] T. L. Gilbert and J. M. Kelly, in Proceedings of the Conference on Magnetism and Magnetic Materials, Pittsburgh, PA, June 14-16, 1955 (American Institute of Electrical Engineers, New York, 1955), pp. 253-263.

[8] T. L. Gilbert, Ph.D. thesis, Illinois Institute of Technology, Chicago, 1956.

[9] T. L. Gilbert, IEEE Trans. Magn. 40, 3443 (2004).

[10] V. Kamberský, Can. J. Phys. 48, 2906 (1970).
[11] V. Kamberský, Czech. J. Phys. B 26, 1366 (1976).

[12] V. Korenman and R. E. Prange, Phys. Rev. B 6, 2769 (1972).

[13] J. Kuneš and V. Kamberský, Phys. Rev. B 65, 212411 (2002).

[14] V. Kamberský, Phys. Rev. B 76, 134416 (2007).

[15] Y. Tserkovnyak, A. Brataas, and G. E. W. Bauer, Phys. Rev. Lett. 88, 117601 (2002).

[16] M. C. Hickey and J. S. Moodera, Phys. Rev. Lett. 102, 137601 (2009).

[17] K. Gilmore, Y. U. Idzerda, and M. D. Stiles, Phys. Rev. Lett. 99, 027204 (2007).

[18] R. Mondal, M. Berritta, and P. M. Oppeneer, Phys. Rev. B 94, 144419 (2016).

[19] R. Mondal, M. Berritta, and P. M. Oppeneer, Phys. Rev. B 98 , 214429 (2018).

[20] R. Mondal, M. Berritta, and P. M. Oppeneer, J. Phys.: Condens. Matter 30, 265801 (2018).

[21] R. Mondal, M. Berritta, K. Carva, and P. M. Oppeneer, Phys. Rev. B 91, 174415 (2015).

[22] R. Mondal, M. Berritta, C. Paillard, S. Singh, B. Dkhil, P. M. Oppeneer, and L. Bellaiche, Phys. Rev. B 92, 100402(R) (2015).

[23] R. Mondal, A. Donges, U. Ritzmann, P. M. Oppeneer, and U. Nowak, Phys. Rev. B 100, 060409(R) (2019).

[24] R. Mondal, A. Donges, and U. Nowak, Phys. Rev. Research 3, 023116 (2021). 
[25] H. Suhl, IEEE Trans. Magn. 34, 1834 (1998).

[26] M.-C. Ciornei, J. M. Rubí, and J.-E. Wegrowe, Phys. Rev. B 83, 020410(R) (2011).

[27] M.-C. Ciornei, Ph.D. thesis, Ecole Polytechnique, Universidad de Barcelona, 2010.

[28] J.-E. Wegrowe and M.-C. Ciornei, Am. J. Phys. 80, 607 (2012).

[29] D. Böttcher and J. Henk, Phys. Rev. B 86, 020404(R) (2012).

[30] R. Mondal, M. Berritta, A. K. Nandy, and P. M. Oppeneer, Phys. Rev. B 96, 024425 (2017).

[31] M. Fähnle and C. Illg, J. Phys.: Condens. Matter 23, 493201 (2011).

[32] M. Fähnle, D. Steiauf, and C. Illg, Phys. Rev. B 84, 172403 (2011).

[33] S. Bhattacharjee, L. Nordström, and J. Fransson, Phys. Rev. Lett. 108, 057204 (2012).

[34] U. Bajpai and B. K. Nikolić, Phys. Rev. B 99, 134409 (2019).

[35] D. Thonig, O. Eriksson, and M. Pereiro, Sci. Rep. 7, 931 (2017).

[36] Y. Li, A.-L. Barra, S. Auffret, U. Ebels, and W. E. Bailey, Phys. Rev. B 92, 140413(R) (2015).

[37] I. Makhfudz, E. Olive, and S. Nicolis, Appl. Phys. Lett. 117, 132403 (2020).

[38] K. Neeraj, N. Awari, S. Kovalev, D. Polley, N. Zhou Hagström, S. S. P. K. Arekapudi, A. Semisalova, K. Lenz, B. Green, J.-C. Deinert, I. Ilyakov, M. Chen, M. Bawatna, V. Scalera, M. d'Aquino, C. Serpico, O. Hellwig, J.-E. Wegrowe, M. Gensch, and S. Bonetti, Nat. Phys. 17, 245 (2020).

[39] S. Giordano and P.-M. Déjardin, Phys. Rev. B 102, 214406 (2020)

[40] S. V. Titov, W. T. Coffey, Y. P. Kalmykov, M. Zarifakis, and A. S. Titov, Phys. Rev. B 103, 144433 (2021).

[41] M. Cherkasskii, M. Farle, and A. Semisalova, Phys. Rev. B 102, 184432 (2020).

[42] M. Cherkasskii, M. Farle, and A. Semisalova, Phys. Rev. B 103, 174435 (2021).

[43] A. M. Lomonosov, V. V. Temnov, and J.-E. Wegrowe, Phys. Rev. B 104, 054425 (2021).

[44] R. Rahman and S. Bandyopadhyay, J. Phys.: Condens. Matter 33, 355801 (2021).
[45] S. V. Titov, W. T. Coffey, Y. P. Kalmykov, and M. Zarifakis, Phys. Rev. B 103, 214444 (2021).

[46] R. Mondal, J. Phys.: Condens. Matter 33, 275804 (2021).

[47] R. Mondal, S. Großenbach, L. Rózsa, and U. Nowak, Phys. Rev. B 103, 104404 (2021).

[48] R. Cheng, X. Wu, and D. Xiao, Phys. Rev. B 96, 054409 (2017).

[49] A. Kamra, R. E. Troncoso, W. Belzig, and A. Brataas, Phys. Rev. B 98, 184402 (2018).

[50] Q. Liu, H. Y. Yuan, K. Xia, and Z. Yuan, Phys. Rev. Materials 1, 061401(R) (2017).

[51] H. Y. Yuan, Q. Liu, K. Xia, Z. Yuan, and X. R. Wang, Europhys. Lett. 126, 67006 (2019).

[52] M. T. Hutchings and E. J. Samuelsen, Phys. Rev. B 6, 3447 (1972).

[53] S. Baierl, J. H. Mentink, M. Hohenleutner, L. Braun, T.-M. Do, C. Lange, A. Sell, M. Fiebig, G. Woltersdorf, T. Kampfrath, and R. Huber, Phys. Rev. Lett. 117, 197201 (2016).

[54] T. Archer, R. Hanafin, and S. Sanvito, Phys. Rev. B 78, 014431 (2008).

[55] T. Archer, C. D. Pemmaraju, S. Sanvito, C. Franchini, J. He, A. Filippetti, P. Delugas, D. Puggioni, V. Fiorentini, R. Tiwari, and P. Majumdar, Phys. Rev. B 84, 115114 (2011).

[56] M. J. Besnus and A. J. P. Meyer, Phys. Status Solidi B 58, 533 (1973).

[57] R. Zhang, R. Skomski, X. Li, Z. Li, P. Manchanda, A. Kashyap, R. D. Kirby, S.-H. Liou, and D. J. Sellmyer, J. Appl. Phys. 111, 07D720 (2012).

[58] S. Alebrand, M. Gottwald, M. Hehn, D. Steil, M. Cinchetti, D. Lacour, E. E. Fullerton, M. Aeschlimann, and S. Mangin, Appl. Phys. Lett. 101, 162408 (2012).

[59] S. Mangin, M. Gottwald, C.-H. Lambert, D. Steil, V. Uhlîr, L. Pang, M. Hehn, S. Alebrand, M. Cinchetti, G. Malinowski, Y. Fainman, M. Aeschlimann, and E. E. Fullerton, Nat. Mater. 13, 286 (2014).

[60] A. Ciuciulkaite, K. Mishra, M. V. Moro, I.-A. Chioar, R. M. Rowan-Robinson, S. Parchenko, A. Kleibert, B. Lindgren, G. Andersson, C. S. Davies, A. Kimel, M. Berritta, P. M. Oppeneer, A. Kirilyuk, and V. Kapaklis, Phys. Rev. Materials 4, 104418 (2020). 